\title{
Desarrollo de la extensión en las universidades estatales costarricenses. Influencias, modelos y desafíos actuales
}

Sandra Chaves Bolaños

schavess@uned.ac.cr

Catherine Lara Campos

clara@uned.ac.cr

Adriana Villalobos Araya

advillalobos@uned.ac.cr
Dirección de Extensión de la Universidad Estatal a Distancia,

Costa Rica.
A 100 años de la Reforma Universitaria de 1918

Desafíos de gestión

RECEPCIÓN: 03/05/17

ACEPTACIÓN FINAL: 10/10/17

\section{Resumen}

Este artículo pretende plasmar el quehacer de las universidades estatales costarricenses en el campo de la extensión universitaria a la luz de algunos acontecimientos históricos a nivel mundial. Con el propósito de que la extensión universitaria en Costa Rica cumpla un rol de transformación social mediante la generación del conocimiento, se analizó la praxis de este pilar de la educación superior en las cinco universidades estatales y se plantean aspectos de mejora para trascender los modelos actuales.
Palabras clave

- Extensión

- Universidad sociedad

- Transformación socia

- Modelos de extensión

\section{Resumo}

Este artigo pretende plasmar as ações das universidades públicas costarriquenhas no campo da extensão universitária à luz de acontecimentos históricos no âmbito mundial. Com o propósito de que a extensão universitária na Costa Rica cumpra um papel de transformação social ao gerar conhecimento, analisou-se a práxis deste pilar da educação superior nas cinco universidades públicas e se formulam aspectos de melhoria para transcender os modelos atuais.

\section{Palavras-chave}

- Extensão

- Universidade-sociedade

- Transformação social

- Modelos de extensão
Para citación de este artículo

Bolaños, S.; Lara Campos, C. Villalobos Araya, A. (2017). Desarrollo de la extensión en las universidades estatales costarricenses. Influencias, modelos y desafíos actuales. . Revista $+E$ versión en línea, 7(7), 122-131. Santa Fe, Argentina: Ediciones UNL. 


\section{Introducción}

El artículo presenta un análisis del desarrollo del quehacer de la extensión en las universidades estatales costarricenses a la luz de hitos históricos acontecidos en el ámbito mundial. La concepción filosófica y metodológica de este pilar académico se ha construido a través del tiempo, con aciertos y desaciertos que deben ser analizados. Este análisis tiene el propósito de presentar planteamientos orientados a la mejora de los procesos y redefinir su accionar de manera que responda a los requerimientos actuales y logre el éxito de las trasformaciones sociales y universitarias. El rol de la extensión como encargada de la vinculación universidad-sociedad conlleva gran responsabilidad. De forma tal que el alcance de una mejor calidad de vida de los habitantes mediante la democratización del conocimiento y la cultura depende en gran medida de cómo se concibe la vinculación, cómo se realiza y a quiénes involucra. Por lo tanto, para dar respuestas a estas interrogantes surge la necesidad de una mirada en retrospectiva de la extensión universitaria.

El artículo corresponde a una investigación descriptiva, puesto que recolecta datos con aspectos relevantes del objeto de estudio. El método utilizado es cualitativo, lo que permite "el desarrollo natural de los sucesos, es decir, no hay manipulación, ni estimulación con respecto a la realidad". Asimismo, tiene un componente interpretativo, el cual "intenta encontrar sentido a los fenómenos en términos de los significados que las personas les otorguen" (Corbetta, 2003, citado por Hernández et al., 2007:9). La investigación se apoya en la recolección de datos históricos y bibliográficos referidos a la extensión universitaria e intenta conocer las expectativas futuras y de desarrollo que pueda alcanzar la extensión a corto y mediano plazo. ${ }^{1}$

El artículo presenta, en primer término, las definiciones institucionales sobre extensión universitaria; en un segundo apartado se desarrollan los modelos de extensión que orientan las prácticas sociales, y se concluye con una serie de reflexiones en relación con el quehacer de la extensión universitaria y sus perspectivas.

\section{Las definiciones institucionales de la extensión en Costa Rica} La extensión universitaria tiene sus inicios en 1790 en Gran Bretaña con la conformación de programas de educación para adultos con miras a atender las necesidades de la Revolución Industrial. Por otra parte, entre 1860 y 1862, en Estados Unidos las universidades desarrollaron un marco filosófico denominado Land-Grant Pilosophy, el cual dio origen a centros de enseñanza dirigidos a campesinos en temas de producción agropecuaria (Cantero, 2006). Estas primeras prácticas evidencian el surgimiento de una labor académica para el fortalecimiento de capacidades productivas y laborales. En el ámbito latinoamericano, las universidades eran consideradas elitistas y manejadas por los intereses de las clases dominantes. Para 1918, y como reacción a las ideas retrógradas de una universidad ligada a la Iglesia Católica y a la oligarquía local, surge en Argentina la Reforma de Córdoba, movimiento que se convierte en referente para las universidades latinoamericanas y cuyo propósito principal fue buscar mayor incorporación de la población a la universidad indistintamente de su condición social, así como el acercamiento de la población a la cultura. Tal y como menciona Tünnermann Bernheim: "La clase media emergente fue, en realidad, la protagonista del movimiento, en su afán de lograr acceso a la universidad" (2008:97).

A partir de 1940, en Costa Rica se establecieron cinco universidades estatales: Universidad de Costa Rica (UCR), fundada en 1940; Instituto Tecnológico de Costa Rica (ITCR), creado en 1971; Universidad Nacional Costa Rica (UNA), creada en 1973; Universidad Estatal a Distancia (UNED), constituida en 1976, y Universidad Técnica Nacional (UTN), establecida en 1988. En cada una de estas universidades, desde su creación, se evidencia en los estatutos orgánicos y documentos oficiales la extensión como un pilar académico de la enseñanza superior pública.

Carlos Tünnermann Berheim (1978), señala el impacto que tuvo la reforma que se da en el país y Centroamérica, en relación con las universidades y la extensión:

"En el área centroamericana, los principales principios reformistas fueron incorporados en las legislaciones universitarias aprobadas a fines de la década de los años 40 en Guatemala, El Salvador, Costa Rica y Panamá y hacia fines de la década del 50 en Honduras y Nicaragua. Sin embargo, desde 1948, con motivo del 'Primer Congreso Universitario Centroamericano' reunido en San Salvador y que dio origen al CSUCA, las Universidades de la región aprobaron una 'Declaración de Principios, Fines y Funciones de la Universidad Contemporánea, y en especial de las Universidades de Centroamérica', en la cual se dice que 'como una forma viva de ideales y actividades, la Universidad debe ser creadora de cultura en los múltiples aspectos de la labor valorativa del hombre, y difusora de la cultura recibida sumada a la riqueza de su propio espíritu creador. Creación y difusión de cultura que sirven en función social a la comunidad'. Más adelante, al referirse a los servicios a la comunidad, la Declaración de Principios agrega: 'La Universidad debe ser activa, y de esta suerte, lo que en ella se enseñe debe traducirse 
en funciones, servicios y prácticas, a cargo de maestros, alumnos y graduados en general. Tales funciones, servicios y practicas deben revertir en beneficio de la comunidad a fin de que la Universidad no sea solo acumulación de cultura y transmisión del saber, sino un sistema de funciones que beneficie a la colectividad de que se nutre'". (1978:104)

En 1949 se realizó el Primer Congreso de Universidades Latinoamericanas en Guatemala, donde se propuso implementar la extensión en dos ramas: la social y la cultural.

En 1957, la Unión de Universidades de América Latina convocó en Santiago de Chile a la Primera Conferencia Latinoamericana de Extensión y Difusión Cultural y en esta se adoptó como concepto de extensión universitaria lo siguiente:

"La extensión universitaria debe ser conceptuada por su naturaleza, contenido, procedimientos y finalidades, de la siguiente manera. Por su naturaleza, la extensión universitaria es misión y función orientadora de la universidad contemporánea, entendida como ejercicio de la vocación universitaria. Por su contenido y procedimiento, la extensión universitaria se funda en el conjunto de estudios y actividades filosóficas, científicas, artísticas y técnicas, mediante el cual se auscultan, exploran y recogen del medio social, nacional y universal, los problemas, datos y valores culturales que existen en todos los grupos sociales. Por sus finalidades, la extensión universitaria debe proponerse, como fines fundamentales proyectar dinámica y coordinadamente la cultura y vincular a todo el pueblo con la universidad". (UDUAL, citado por Tünnermann Berheim, 2000:s/n)

A partir de este concepto se asumió que mediante la extensión se debía procurar el desarrollo social, espiritual e intelectual de las naciones mediante la propuesta de soluciones a problemas de interés general y la proyección a la comunidad. Se incorporaron a la universidad tareas de extensión y difusión al quehacer universitario desde la perspectiva de la proyección comunal y predominó el criterio de entrega de la cultura a los menos favorecidos pero "en una sola vía, desde la universidad, depositaria del saber y la cultura, al pueblo, simple destinatario de esa proyección y al cual se supone incapaz de aportar nada valioso" (5).

Ese concepto basado en la difusión cultural se mantuvo durante los años 60 y 70 e incorporó algunos programas de acción social, especialmente en el campo de la salud, la alfabetización y educación de adultos, así como la asesoría jurídica.

En los años 90 se destacaron otros avances, como la publicación del Programa Nacional de Extensión de la Cultura y los Servicios, en 1992, por la Asociación Nacional de Universidades e Instituciones de Educación Superior (ANUIES). Así como la elaboración de Subprogramas Nacionales en las áreas de divulgación científica, tecnológica, social y artística. ANUIES, citado por Serna (2016) define a la extensión como:

"Una función que tiene como finalidad el hacer participar de los beneficios de la educación y la cultura nacional y universal a todos los sectores de la sociedad tratando de incidir en su integración y transformación mediante la difusión y divulgación, promoción y servicios del conocimiento científico, tecnológico, artístico y humanístico". (1991:5)

Las anteriores definiciones, concuerdan con la construcción de la política institucional en temas de extensión y acción social en las universidades costarricenses. No obstante, en el Cuadro $N^{\circ} 1$ se visualiza el carácter identitario de cada universidad. Por otra parte, el Consejo Nacional de Rectores (CONARE), en 2017, define la extensión y acción social, como el contacto con la sociedad para participar en el proceso de cambio, enriqueciéndola con valores y conocimientos en procura de conservar las manifestaciones culturales. Dicha instancia juega un papel fundamental en la articulación de las universidades estatales, lo cual permite la construcción de una planificación conjunta de la educación superior, implementa procesos integrados que optimizan la calidad, maximizan los recursos e impactan positivamente en el desarrollo del país. De esta forma, se logra una sinergia entre las cinco universidades que lo conforman, aprovechando las fortalezas y potencialidades de cada una.

Las universidades estatales costarricenses son miembros del Sistema Centroamericano de Relación Universidad-Sociedad (SICAUS). Este sistema universitario regional de la Confederación Universitaria Centroamérica es organismo adscrito al Consejo Superior Universitario Centroamericano (CUSCA).

EI SICAUS es un ente de naturaleza académica y técnica integrado por los vicerrectores y directores de Extensión de las universidades miembros del CSUCA. Su propósito es interactuar con los diversos sectores de la sociedad a fin de contribuir al desarrollo de procesos de integración y desarrollo regional, fortaleciendo la relación universidad-sociedad.

De acuerdo con lo establecido en la XXIV Asamblea Regional realizada en Managua, Nicaragua, en junio de 2017, se reconoce la extensión/vinculación/proyección social/acción social universitaria como una función académica esencial e integradora del quehacer de la universidad pública centroamericana y latinoamericana, y junto a la investigación científica y la docencia, a partir de este planteamiento, las universidades desarrollan procesos conjuntos orientados a la formulación y ejecución de proyectos que contribuyan al bienestar de las comunidades. 


\section{Cuadro $\mathbf{N}^{\circledR}$ 1. Conceptualización de la extensión o acción social en las universidades estatales de Costa Rica}

\begin{tabular}{|c|c|c|}
\hline Universidad & Instancia rectora en la materia & Concepción de la extensión y acción social \\
\hline UCR & Vicerrectoría de Acción Social & $\begin{array}{l}\text { "Constituye el vínculo principal entre la Universidad de Costa Rica y el país por medio de sus programas de } \\
\text { divulgación, extensión y trabajo comunal” (UCR, 1980). }\end{array}$ \\
\hline ITCR & $\begin{array}{l}\text { Vicerrectoría de Investigación } \\
\text { y Extensión Universitaria }\end{array}$ & $\begin{array}{l}\text { "La investigación y la extensión son actividades fundamentales del Instituto orientadas a la generación, adaptación, } \\
\text { incorporación y comunicación del conocimiento tecnológico, científico, educativo y sociocultural” (ITCR, 2010). }\end{array}$ \\
\hline UNA & Vicerrectoría de Extensión & $\begin{array}{l}\text { "Constituye una tarea por medio de la cual la Universidad se interrelaciona con la comunidad nacional, de manera } \\
\text { crítica y creadora. Por medio de ésta proyecta a la sociedad los productos del quehacer académico, lo que } \\
\text { permite que se redimensione y enriquezca al percibir las necesidades de la sociedad" (Villalobos, 2017). }\end{array}$ \\
\hline UNED & $\begin{array}{l}\text { Dirección de Extensión } \\
\text { Universitaria }\end{array}$ & $\begin{array}{l}\text { Constituye "una relación dialéctica universidad-sociedad que contempla los procesos de educación no formal } \\
\text { desde la UNED, para incidir en las condiciones de vida de las poblaciones, especialmente aquellas que por } \\
\text { razones económicas, sociales, geográficas, culturales, etarias, de discapacidad, de género, requieren de } \\
\text { oportunidad para una inserción real y equitativa en la sociedad" (UNED, 2013). }\end{array}$ \\
\hline UTN & $\begin{array}{l}\text { Vicerrectoría de Extensión } \\
\text { y Acción Social }\end{array}$ & $\begin{array}{l}\text { Contribuye a "emprender proyectos o acciones de extensión viables que faciliten las vías para generar prosperidad } \\
\text { económica, desarrollo, más empleo y la continuidad en el tiempo necesaria para cultivar en las personas, la } \\
\text { convicción de sentir pasión por su trabajo y la ilusión de ser forjador de su propio futuro, integrándose a la } \\
\text { sociedad y vinculándose al mercado de trabajo de forma más autónoma y competitiva" (UTN, 2015). }\end{array}$ \\
\hline
\end{tabular}

Fuente: elaboración propia a partir de información recopilada en las universidades estatales costarricenses.

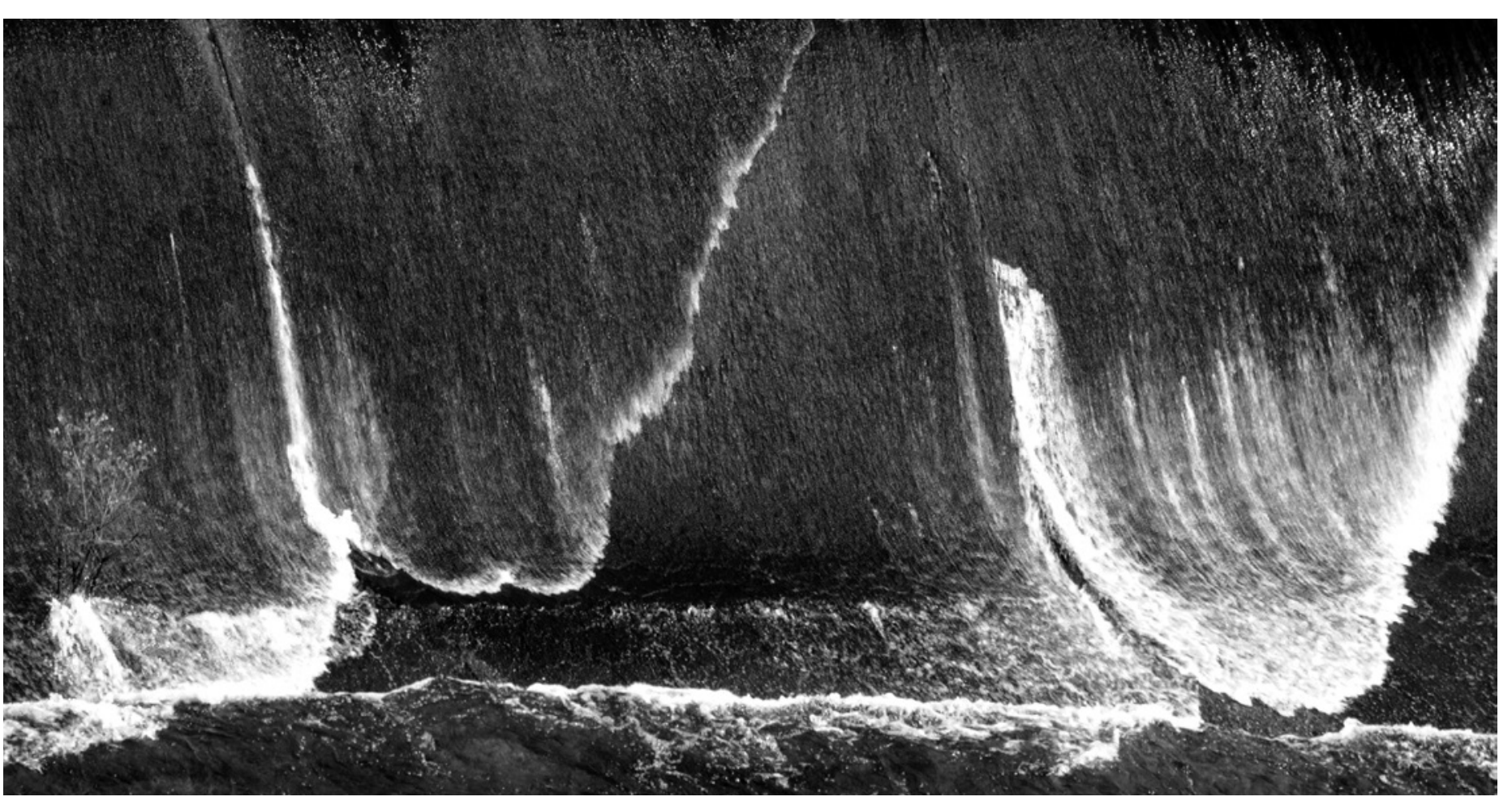

(c) Laura Hormaeche 


\section{Los modelos de extensión que orientan las prácticas sociales universitarias en Costa Rica}

La concepción metodológica de la extensión universitaria debe mantenerse en constante evolución, de forma tal que se adapte a una sociedad con características y necesidades cambiantes. Por lo tanto, es fundamental una mirada crítica a los modelos de extensión implementados alrededor del mundo y un análisis introspectivo de las prácticas llevadas a cabo en las universidades estatales de Costa Rica.

De acuerdo con Serna (2007), se han identificado como modelos de extensión universitaria el altruista, el divulgativo, el concientizador, el vinculatorio empresarial y la extensión como subsistema institucional. Cada uno de estos se implementa en mayor o menor medida en las cinco universidades públicas de Costa Rica.

El altruista promueve la acción humanitaria de académicos y estudiantes a favor de poblaciones vulnerables como retribución al pueblo que cubre los costos de la educación superior. Algunos ejemplos de su accionar son las brigadas de salud, bufetes jurídicos, capacitaciones, entre otros. En el caso de Costa Rica, se brinda servicio social mediante voluntariado y trabajo comunal universitario, procesos que han incidido en la solución de problemas de la población y contribuyen a la práctica académica y formación de compromiso social de los futuros graduados.

El divulgativo adapta la información para transferir a la sociedad conocimientos y expresiones culturales mediante el uso de medios de comunicación. Es un modelo que fue criticado por no permitir una comunicación universidad-sociedad en doble vía, pudiendo establecer relaciones de poder en términos de la invasión cultural y la imposición de valores a los individuos.

En el ámbito costarricense se utiliza el modelo divulgativo y juega un papel fundamental en el acceso a la información y conocimiento de la sociedad. Con el desarrollo tecnológico, los medios de comunicación se vuelven más amigables al permitir espacios de intercambio sincrónico y asincrónico con los sectores sociales. Si bien es cierto que las cinco universidades públicas incursionan en este modelo, la UNED por su condición de universidad a distancia ha logrado un reconocimiento en el uso de las tecnologías de la información y comunicación (TIC) para el desarrollo de la educación superior.

El modelo concientizador consiste en compartir la educación y la cultura de forma liberadora, promoviendo el análisis crítico y una acción eficaz. Sin embargo, Serna identifica obstáculos para su aplicación:

"pierde de vista la existencia de muchos factores que no dependen de la voluntad o del grado de compromiso de los universitarios que realizan labores de Extensión. Esos factores van desde la vertical estratificación de la burocracia gubernamental y universitaria hasta la dependencia de amplios sectores populares al paternalismo atávico ejercido por el Estado". (2016:8)
Uno de los saltos cualitativos de las universidades costarricenses en la práctica de la extensión, ha sido la implementación de procesos educativos continuos, multi y transdisciplinarios orientados a la transformación social. Estos emplean metodologías que permiten un diálogo de saberes y una construcción conjunta del conocimiento. La participación activa de los beneficiarios se traduce en capacidades de autogestión de su propio desarrollo. Asimismo, las universidades juegan un rol articulador de actores sociales, ya que en estos procesos educativos no solo forman parte académicos, estudiantes y comunidades, sino, también ha logrado integrar a instituciones públicas, empresas privadas, organizaciones del tercer sector y otros.

El modelo vinculatorio empresarial permite la articulación entre los sectores empresariales y las universidades con el fin de generar y transmitir conocimientos aplicables al sector productivo. Sin embargo, no deben descuidarse otras demandas sociales por privilegiar las necesidades empresariales.

En el ámbito costarricense, la relación universidad-empresa comenzó a fortalecerse y a ser redituable facilitando diversos servicios como: cursos, asesorías, investigaciones y desarrollo tecnológico, incubadora de empresas, entre otras. Esta relación genera y maximiza recursos en beneficio de ambos sectores y realimenta el quehacer académico para la mejora curricular. El modelo denominado "la extensión como subsistema institucional", induce a un análisis de la necesidad de interrelación entre la extensión, investigación y docencia para el funcionamiento adecuado del sistema universitario. Como lo explica la Teoría general de sistemas, la relación entre las partes genera sinergias que trasciende el accionar de cada una de estas. Por lo tanto, la potencialidad de la extensión universitaria debe enmarcarse en relaciones con actores sociales, pero también, universitarios como los estudiantes, docentes e investigadores, para que de manera conjunta se identifiquen necesidades, se seleccionen problemas y se construyan alternativas de solución; realimentando así, todo el quehacer académico (Serna, 2016).

La interrelación entre los pilares de la educación superior, aún representa un reto en las universidades del país, ya que, si bien es cierto, la política institucional la identifica como una función sustantiva, existe un rezago en la dotación de recursos y posicionamiento frente a la investigación y la docencia. Esto se visualiza en la distribución de cargas académicas, el presupuesto asignado, la falta de incentivos en el desarrollo profesional y en el caso de la UNED existe una diferenciación jerárquica puesto que se mantiene como dirección. Otra de las causas es la falta de comunicación y espacios de encuentro para la reflexión y planificación conjunta.

Una mirada retrospectiva sobre el quehacer extensionista permite identificar aspectos a mejorar, así como también, rescata aportes de cada modelo utilizado. Con el propósito de lograr la resignificación de la extensión, conviene que las universidades continúen: 


\title{
G6
}

\author{
Uno de los saltos cualitativos de las \\ universidades costarricenses en la práctica \\ de la extensión, ha sido la implementación \\ de procesos educativos continuos, multi \\ y transdisciplinarios orientados a la \\ transformación social
}

- prestando servicios a la sociedad y prioritariamente, a aquellas poblaciones en condición de vulnerabilidad

- manteniendo la divulgación de la información y nuevos conocimientos, bajo un enfoque de respeto al saber tradicional, los valores y cultura,

- sosteniendo procesos de construcción participativa del conocimiento mediante un diálogo de saberes que permita la autogestión, el análisis crítico y la implementación de soluciones acordes a la realidad social.

- la vinculación con actores sociales entendidos como: organismos internacionales, organizaciones sin fines de lucro, organizaciones comunales, instancias gubernamentales, empresa privada y sociedad civil, con el fin de contribuir al buen vivir de una nación y al mejoramiento continuo de la educación superior.

Las universidades estatales costarricenses en materia de extensión han seguido las corrientes mundiales, adaptándose a las diversas modalidades utilizadas para el trabajo con las comunidades. Al respecto, Saviani (1981) afirma: "corresponde a la universidad socializar sus conocimientos, extendiéndolos a la comunidad y convirtiéndose, así, en una fuerza viva capaz de elevar el nivel cultural general de la sociedad" (citado por Duarte y Alves, 2015:15).

Ante este panorama, surge la importancia de la pertinencia de los servicios ofrecidos por la universidad, los cuales deben estar sustentados en el compromiso social. Para definir la pertinencia se analiza el cumplimiento de expectativas y exigencias sociales con acciones comprometidas, relevantes y oportunas. Las universidades que atienden las necesidades de su contexto y garantizan su presencia en la resolución de los problemas emergentes, aseguran su subsistencia como institución capaz de producir saberes de calidad, de espacios de discusión democrática y participativa y de respuestas de significación social tendientes a aportar para una mejor calidad de vida de los habitantes (Pérez, 2007).
Para garantizar la pertinencia, las universidades deben afianzar su relación dialéctica con la sociedad, enfocándose no solo en la venta de servicios sino en una extensión con compromiso social. Según palabras de Llorens:

"la extensión debe ser el espacio, el ambiente y el lenguaje para la comunicación entre la universidad y la sociedad, así como entre los universitarios, un espacio común, necesario para el enriquecimiento del saber, el disfrute y la crítica de la cultura en sus manifestaciones éticas, materiales, estéticas, científicas y tecnológicas". (1992:7)

Se hace necesaria entonces, la creación de competencias en el estudiantado, de manera que se les permita la puesta en práctica del conocimiento adquirido, pero no desde la perspectiva de poseedores de la verdad, si no desde la creación conjunta y el aprendizaje mutuo. Es por esto que la extensión debe avanzar para convertirse en un eje transversal del currículo, de manera que esté implícita siempre y se convierta en el medio de comprensión de la realidad.

En el siglo XXI, los paradigmas de formación, integración y calidad que debe asumir la universidad, así como la aceleración de los procesos (demográficos, tecnológicos, urbanos, sociales, ambientales, productivos, económicos y otros), hacen de la extensión un vehículo importante no solo de transferencia, sino de diálogo de saberes y reflexión (Flores et al., 2011). Hoy quienes demandan el cumplimiento de la función extensionista, requieren de una mediación pedagógica que induzca al análisis crítico, al empoderamiento y a la participación en la toma de decisiones, por lo tanto, es necesario promover relaciones y estrategias de trabajo adaptadas a las circunstancias históricas o nuevas realidades (Pérez, 2007).

Dicha actividad académica no debe partir de una visión administrativa sino entenderse en términos de la necesidad de "aprender a aprender", "aprender a emprender", "aprender a convivir" y "aprender a ser". En la medida en que las universidades 
asuman la extensión, contribuirán a la educación de jóvenes, de individuos de todas las edades, formando seres humanos capaces de descubrir y reafirmar día a día su saber, de mirar críticamente la realidad y de participar en los procesos de transformación social (Fresán, 2004).

Sin embargo, no podemos quedarnos en el mero recuento de lo actuado, el análisis del camino recorrido tiene que llevarnos a la reflexión sobre el papel que desde las universidades debe asumir la extensión. Desde esa perspectiva, se espera de la extensión un accionar planificado, ordenado y con incidencia positiva en la realidad social. De forma tal que se convierta en el medio para la comprensión y búsqueda de respuestas a problemáticas específicas en las poblaciones.

Mucho se ha hecho pero el camino por recorrer es largo; los modelos de extensión actuales pueden ser mejorados. Es necesario el análisis del quehacer pero solo desde la perspectiva de la academia, los actores sociales tienen mucho que aportar en esta relación dialéctica para la producción de nuevos conocimientos, y desde esa perspectiva el diálogo de saberes, los espacios de discusión y reflexión sobre los valores que deben prevalecer en el

Cuadro $\mathrm{N}^{\circ}$ 2. Criterios y acciones en desarrollo y por desarrollar de las universidades estatales de Costa Rica

\begin{tabular}{|c|c|c|}
\hline Criterios de los trabajos de extensión & Acciones en desarrollo o desarrolladas & Acciones por desarrollar \\
\hline Excelencia y calidad. & $\begin{array}{l}\text { Creación de espacios de discusión y reflexión de las } \\
\text { problemáticas de la sociedad costarricense. } \\
\text { Análisis de pertinencia y excelencia de los procesos } \\
\text { académicos. }\end{array}$ & $\begin{array}{l}\text { Fomento del diálogo de saberes entre extensión, } \\
\text { comunidades y actores locales para generar acciones } \\
\text { pertinentes que repercutan en un mejoramiento de la calidad. } \\
\text { Reconocimiento en puntajes del ascenso en régimen de } \\
\text { carrera profesional de trabajos de extensión y acción social, } \\
\text { para promover la participación de los académicos en este } \\
\text { quehacer sustantivo. }\end{array}$ \\
\hline $\begin{array}{l}\text { Carácter innovador y productor de } \\
\text { conocimiento. }\end{array}$ & $\begin{array}{l}\text { Implementación de proyectos pertinentes orientados } \\
\text { al bienestar de las comunidades mediante fuentes de } \\
\text { financiamiento adicionales para promover el desarrollo } \\
\text { de proyectos. }\end{array}$ & Incremento de la divulgación y difusión del quehacer. \\
\hline Efecto demostrativo. & $\begin{array}{l}\text { Ampliación de espacios de capacitación en temas de } \\
\text { gestión y sistematización. }\end{array}$ & $\begin{array}{l}\text { Definición y vinculación de los procesos de acreditación con } \\
\text { la extensión. }\end{array}$ \\
\hline Mejora continua. & $\begin{array}{l}\text { Desarrollo de sistemas de información: gestión de } \\
\text { proyectos, que buscan la calidad de datos con fines } \\
\text { estadísticos y la generación de información para la } \\
\text { toma de decisiones de las autoridades pertinentes. }\end{array}$ & $\begin{array}{l}\text { Diseño de procedimientos para formulación, evaluación y } \\
\text { control de los procesos en todas las unidades académicas. }\end{array}$ \\
\hline $\begin{array}{l}\text { Vinculación con la docencia y la } \\
\text { investigación. }\end{array}$ & $\begin{array}{l}\text { Desarrollo de la extensión y acción social por todas las } \\
\text { unidades académicas de las universidades. }\end{array}$ & $\begin{array}{l}\text { Creación de sistemas de monitoreo, seguimiento y } \\
\text { evaluación en todos los procesos e instancias. }\end{array}$ \\
\hline Procesos de información. & Creación de medios de divulgación del quehacer. & $\begin{array}{l}\text { Maximización de la divulgación de los procesos de extensión } \\
\text { universitaria. }\end{array}$ \\
\hline
\end{tabular}

Fuente: elaboración propia a partir de información recopilada en las universidades estatales costarricenses. 
ejercicio de la extensión como actividad académica y que pueden conducir a acciones demostrativas de lo que es posible llevar a la práctica mediante la ejecución de proyectos de acción social. No debe olvidarse que en este proceso tendrá un papel fundamental la sistematización de la gestión como un medio para preservar y replicar el conocimiento.

Reflejo de lo anterior es lo que las universidades estatales de Costa Rica han venido trabajando a lo largo de más de 40 años y que puede resumirse en el Cuadro $\mathrm{N}^{\circ} 2$.
En la actualidad, las universidades estatales costarricenses han desarrollado líneas de trabajo como mecanismos de desarrollo en atención a las necesidades comunales e institucionales (ver Cuadro $\mathrm{N}^{\circ} 3$ ).

Todas estas líneas de trabajo tienen como común denominador los actores involucrados, tales como: docentes, estudiantes, instituciones, comunidades, grupos organizados, personal académico y administrativo de las universidades, en procura del mejoramiento del país.

\section{Cuadro $\mathbf{N}^{\circ}$ 3. Líneas de trabajo de las universidades estatales de Costa Rica}

\begin{tabular}{|c|c|}
\hline Línea de trabajo & Actividades \\
\hline 1. Servicio social a favor de personas de escasos recursos. & $\begin{array}{l}\text { - Trabajo Comunal Universitario. } \\
\text { - Voluntariado. Ejemplo: Brigadas de salud y bufetes jurídicos. } \\
\text { - Cursos para población vulnerable. }\end{array}$ \\
\hline
\end{tabular}

3. Actividades deportivas y recreativas.

4. Promoción de diferentes expresiones artísticas y culturales.

5. Difusión masiva de conocimientos a través de los múltiples medios producto de las nuevas tecnologías.

6. Actualización y capacitación permanente en educación no formal.

7. Desarrollo comunal orientados a la autogestión participativa y organización comunitaria.

8. Venta de servicios en innovación y transferencia tecnológica.
- Proyectos de extensión docente.

- Procesos y actividades de las Escuelas vinculadas al deporte.

- Unidades de Bienestar Estudiantil, las cuales fomentan las actividades deportivas y recreativas.

- Proyectos o programas de extensión cultural.

- Se utilizan boletines digitales, portal web, libros, audiovisuales, radio, canales de televisión.

- Videoconferencias, cursos en línea.

- Proyectos de extensión docente.

- Educación continua, cursos de capacitación, seminarios y congresos de actualización académica y científica, entre otros.

- Trabajos comunales universitarios y proyectos de extensión universitario e interuniversitario orientado a diversas problemáticas, como salud, educación, vivienda, alimentación, saneamiento, trabajo, producción a nivel de micro y pequeños emprendimientos, entre otros.

- Venta de servicios por medio de consultorías y cursos a la medida.

Fuente: elaboración propia a partir de información recopilada en las universidades estatales costarricenses. 


\section{Reflexiones finales}

La construcción histórica de la extensión universitaria permitió identificar que su teorización, política, y praxis debe mantenerse en constante evolución para lograr adaptarse a las demandas y necesidades sociales. En sus inicios se planteaba como una ayuda de la universidad a un sector social desfavorecido mediante capacitaciones aisladas o bien como servicios de asesoría que involucraban a extensionistas y estudiantes. Así también, se visualizó como la herramienta para trasladar conocimiento e información desde una posición de superioridad.

A partir de un análisis crítico, se planteó una concepción de educación sustentada en el diálogo de saberes y la construcción de una sociedad más justa y equitativa, para lo cual es importante la promoción de programas multi y transdisciplinarios. La articulación de aliados estratégicos, como organizaciones de base comunal, empresa privada, institucionalidad pública, sociedad civil, organizaciones del tercer sector, entre otras, permite la maximización de los recursos y el desarrollo de procesos sostenibles que contribuyan en la transformación social. La extensión universitaria debe mantener la interacción con

\section{Referencias bibliográficas}

Cantero, C. (2006). El concepto de la extensión universitaria a lo largo de la historia. España: Universidades Públicas Andaluzas. Recuperado de: http://actividades. uca.es/atalaya/atalayaproductos/concepto (consultado el 11/09/2015).

Duarte, L. y Alves, L. (2015). La gestión de la extensión universitaria una nueva sinergia entre los tres pilares de la educación superior universitaria. Invenio: Revista de Investigación Académica, 18(34) 2015: 9-22. Recuperado de: https://dialnet. unirioja.es/servlet/articulo?codigo=5132257 (consultado el 01/03/2017). Hernández, R.; Fernández, C. y Baptista, P. (2007). Metodología de la Investigación. México: Ultra.

Flores, D.; Narváez, J. y González, M. (2011). Principios ontológicos, teleológicos, praxológicos, axiológicos, de la extensión universitaria. [Mensaje en un blog]. Recuperado de http://extension-postgrado-unellez.blogspot.com.ar/2011/11/principios-ontologicos-teleologicos.html

Fresán, M. (2004). La extensión universitaria y la universidad pública. Reencuentro, 39, 47-54. Distrito Federal de México: Universidad Autónoma Metropolitana Unidad Xochimilco. Recuperado de: http://www.redalyc.org/pdf/340/34003906. pdf (consultado el 16/04/ 2017).

Llorens, L. (1992). Planeación y extensión Universitaria. Recuperado de : http:// publicaciones.anuies.mx/pdfs/revista/Revista81_S1A4ES.pdf (consultado el 24/03/2016)

Instituto Tecnológico de Costa Rica (ITCR) (2010). Estatuto Orgánico del Instituto Tecnológico de Costa Rica. Recuperado de: http://www.itcr.ac.cr/reglamentos/ Consultas/consultarR1.asp? $\mathrm{n}=419$ (consultado el 01/03/2016).

Pérez, L. (2007). Pertinencia y extensión universitaria en el contexto de la nueva la docencia y la investigación para potenciar sus impactos y redimensionar el quehacer académico. No obstante, representa un reto la incorporación de la extensión como eje transversal del currículo, principalmente en instituciones de educación a distancia, por lo cual, se recomienda abordar este tema en futuras investigaciones.

Se hace necesario, además, fomentar el diálogo de saberes entre extensión, comunidades y actores locales para generar acciones pertinentes que repercutan en un mejoramiento de la calidad. Así como reconocer en puntajes el ascenso en régimen de carrera profesional de trabajos de extensión y acción social para promover la participación de los académicos en este quehacer sustantivo. Definir y vincular los procesos de acreditación, incrementar la divulgación y difusión del quehacer, así como el diseño, la formulación de procedimientos, la evaluación y el control de los procesos de extensión en todas las unidades académicas de las universidades es otro de los retos importantes que deben ser atendidos a mediano plazo para garantizar la pertinencia y calidad del quehacer extensionista. universidad Cubana. Recuperado de: http://cvi.mes.edu.cu/peduniv/index.php/ peduniv/article/viewFile/395/386 (consultado el 16/09/2017).

Serna, G. (2007). Misión social y modelos de extensión universitaria: del entusiasmo al desdén. Revista Iberoamericana de Educación, (14), 3-4. Recuperado de: http://rieoei.org/deloslectores/1662Aquiles.pdf (consultado el 01/03/16).

Serna, G. (2016). "Modelo de Extensión Universitaria en México". Recuperado de http://www.sld.cu/galerias/pdf/sitios/williamsoler/extension_universitaria.pdf (01/03/16)

Universidad de Costa Rica (UCR) (1980). Reglamento de la Vicerrectoría de Acción Social. La Gaceta Universitaria IV. Recuperado de: http://cu.ucr.ac.cr/normativ/ vicerrectoria_accion_social.pdf

Tünnermann Bernheim, C. (1978). El nuevo concepto de extensión universitaria y difusión cultural y su relación con las políticas de desarrollo cultural en América Latina. Anuario de Estudios Centroamericanos, 4, 93-126.

— (2000). El nuevo concepto de la extensión universitaria. Mimeo. Universidad Michoacana de San Nicolás de Hidalgo Morelia, Michoacán, México, 19 al 23 de noviembre. Recuperado de: http://mokana.udea.edu.co/portal/page/portal/ BibliotecaPortal/InformacionInstitucional/Autoevaluacion/SistemaUniversitarioExtension/NuevoConceptoExtensionUniversitaria-CarlosTunnermann.pdf (consultado el 24/03/2016).

(2008). Noventa años de la Reforma Universitaria de Córdoba

(1918-2008). Buenos Aires: Consejo Latinoamericano de Ciencias Sociales. Recuperado de: http://bibliotecavirtual.clacso.org.ar/ar/libros/grupos/reforTun/ reforTunn.pdf (consultado el 24/03/2017). 


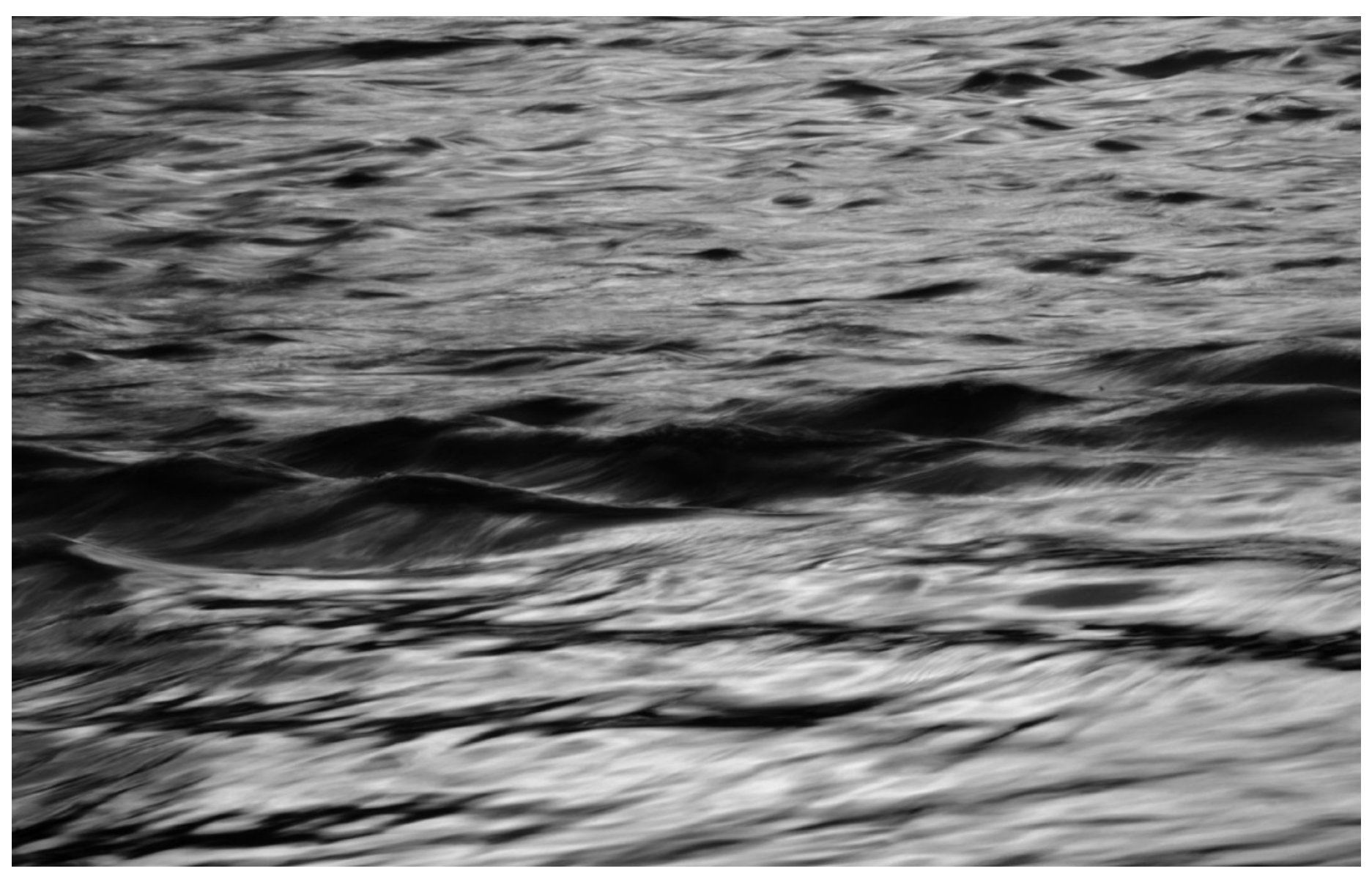

(c) Laura Hormaeche 